\title{
Dementia risk assessment tools: an update
}

\author{
Eugene Yee Hing Tang ${ }^{*}, 1,2$, Louise Robinson ${ }^{1,2}$ \& Blossom Christa Maree Stephan ${ }^{1,2}$ \\ ${ }^{1}$ Institute of Health \& Society, Newcastle University, Baddiley-Clark, Richardson Road, Newcastle upon Tyne, NE2 4AX, UK \\ ${ }^{2}$ Newcastle University Institute of Ageing, Newcastle University, Campus for Ageing \& Vitality, Newcastle upon Tyne, NE4 5PL, UK \\ * Author for correspondence: Tel.: +44 (0)191 208 8758; e.y.h.tang@newcastle.ac.uk
}

"Given the fear and stigma surrounding dementia [16], it is important to seek the views of patients and their families before implementing risk assessment in clinical practice to assist with preventive treatment and future planning."

First draft submitted: 1 September 2017; Accepted for publication: 20 September 2017; Published online: 21 November 2017

Keywords: Alzheimer's disease $\bullet$ dementia $\bullet$ risk factors

In the absence of effective treatments for dementia there has been an international focus towards risk reduction similar to other branches of medicine for example, cardiovascular disease [1]. It has been suggested that around a third of Alzheimer's disease (AD) cases [2] and about a quarter to a third of dementia cases [3] could be prevented through the modification of key risk factors linked to health and lifestyle with examples including low educational attainment and physical inactivity. Some of these factors have been incorporated into models to predict an individual's risk of future dementia. However, previous systematic reviews have found that although some risk tools predict dementia with reasonable accuracy, none are currently recommended for use in clinical settings [4,5]. Since the last systematic review in 2015 there have been further models published. Therefore, the aim of this editorial is to provide an update on new developments in the dementia risk prediction modeling literature.

\section{Current developments in dementia risk prediction modeling}

Recent updates include: development of new genetic risk scores incorporating non-apolipoprotein (APOE) risk genes that are associated with incident $\mathrm{AD}$ [6]; development of a United Kingdom (UK)-based model, incorporating variables that are easily accessible in primary care [7] and testing of model size reduction and incorporating simple variables to reduce the cost/expertise needed for dementia risk score calculation [8]; extension of usage of risk scores into the clinical trial setting; and qualitative assessment of dementia risk reduction. A summary of each of these developments is included below.

\section{Genetic risk scores}

Previous genetic risk scores have assessed the benefits of using APOE e4 and non-APOE e4 genes (PICALM and $C L U)$ to improve predictive models for incident $\mathrm{AD}$ [9]. There have been further models based on genetic risk scores produced since then. One example is a genetic risk score developed in 2016 that used common genetic variants associated with $\mathrm{AD}$ [6]. The authors observed that the aggregate measure of single nucleotide polymorphisms was more significantly associated with incident $\mathrm{AD}$ even without the inclusion of $A P O E$ e4 [6]. The authors assessed a risk model that incorporated age, sex, education and $A P O E$ in risk prediction after 7-year follow-up and found that when the genetic risk score was added to the risk model there was a small improvement in discrimination [6]. These scores could be used in trials to include those found to be at risk but asymptomatic from the disease.

\section{Reducing model complexity}

Although no economic analysis has been previously undertaken, a key criticism of past models was that they often contain resource intensive (in terms of data collection) and costly (in terms of equipment and expertise needed) variables reducing feasibility of implementation. Four studies have focused on reducing model calculation cost and complexity. They first developed a score estimating of 5-, 10- and 20-year dementia risk and focused specifically on risk factors (including age, marital status, body mass index (BMI), stroke, diabetes, ischemic attack 
and cancer) that could be obtained from patient health record data in the UK [7]. The score's predictive accuracy was similar to other studies with the additional benefit of not requiring specialized testing or procedures such as brain imaging [7]. A second study found that data from magnetic resonance imaging does not improve discriminatory performance beyond a model using demographic, cognitive, lifestyle, physical function, health and genetic variables to predict dementia [8]. A third study, using data derived from a primary care database (including age, sex, social deprivation, smoking, BMI, heavy alcohol use, antihypertensive drugs, diabetes, stroke/transient ischemic attack, atrial fibrillation, aspirin and depression) was able to predict 5-year risk of recorded dementia diagnosis in individuals of 60-79 years of age with reasonable accuracy [10]. A fourth study developed a model, which avoided the use of the more 'traditional' vascular dementia risk variables such as blood pressure and cholesterol and instead focused on educational and occupational attainment (i.e., factors important for cognitive reserve) [11]. By utilizing education, factors associated with occupation (e.g., work environment, nature and physicality of work) with and without age, the risk score could predict cognitive functioning and dementia risk 20-40 years later [11]. A further benefit beyond even the advantage of primary care data accessibility is that the model remained accurate even when based on variables that could be self-reported. Such a model is a lot more accessible to the general public than those, that for example require additional blood tests, blood pressure measurements and brain imaging.

\section{Use of risk score in clinical trials}

The Cardiovascular Risk Factors, Aging and Incidence of Dementia (CAIDE) risk score that includes age, hypertension, hypercholesterolaemia, physical inactivity, obesity and educational level [12] and has shown reasonable external validity [13], was incorporated into the Finnish Geriatric Intervention Study to Prevent Cognitive Impairment and Disability (FINGER) trial. The trial found that a multimodal intervention consisting of diet, exercise, cognitive training and vascular risk monitoring could maintain or even improve cognitive functioning in an at-risk population based on the CAIDE score [14]. The CAIDE score has also been developed into an App, which has been found to motivate participants into modifying their risk factors [15]. These studies demonstrate the translatability of the CAIDE risk score and also how it (and possibly other dementia risk scores) could be used clinically in the future.

\section{Patient \& public perceptions of dementia risk reduction}

Given the fear and stigma surrounding dementia [16], it is important to seek the views of patients and their families before implementing risk assessment in clinical practice to assist with preventive treatment and future planning. If patients and their families struggle to understand the outcome of risk assessment for less-stigmatized diseases such as cardiovascular disease [17], then it is even more imperative that, if risk assessment is used for dementia in either the whole or at-risk populations, strategies are developed that ensure that the process is person focused and not disease focused and that there are pathways in place for clinicians to help deal with a positive screening result. There has been a recent attempt to assess perceptions of dementia risk reduction in older and middle-aged adults in a focus group study [18]. The authors found that there was a lack of knowledge about dementia risk factors [18]. The lack of knowledge on dementia risk factors was also seen to be the main barrier for behavioral and lifestyle changes [18]. If risk models incorporating modifiable risk factors for dementia are used, then there needs to be greater promotion and education around dementia risk factors so that at-risk individuals are motivated to reduce their risk of future dementia. Future work could look at the patient and public's views on dementia risk scores to clarify what challenges clinicians may face if they wish to implement risk models into a clinical setting.

\section{Conclusion}

Dementia risk prediction is now beginning to expand beyond just model development. There are examples of trying to ensure that any risk prediction model is clinically appropriate by streamlining the resources required to use the model as well as utilizing them in trials. Future risk models will need to be not only resource efficient but also cost-effective. Future studies are needed to assess how the risk variables interact together to increase an individual's risk of future dementia. By understanding this interplay in more detail, future trials could look to develop multidimensional personalized interventions. From a clinical perspective, this will also allow for more personalized medicine in order to reduce an individual's future risk of dementia.

Financial \& competing interests disclosure

E Tang is supported by a National Institute for Health (NIHR) Doctoral Research Fellowship (DRF-2015-08-006). L Robinson is supported by an NIHR professorship (NIHR-RP-011-043) and an NIHR Senior Investigator Award. The authors have no other relevant 
affiliations or financial involvement with any organization or entity with a financial interest in or financial conflict with the subject matter or materials discussed in the manuscript apart from those disclosed.

No writing assistance was utilized in the production of this manuscript.

\section{References}

1. Baumgart M, Snyder HM, Carrillo MC, Fazio S, Kim H, Johns H. Summary of the evidence on modifiable risk factors for cognitive decline and dementia: a population-based perspective. Alzheimers Dement. 11(6), 718-726 (2015).

2. Norton S, Matthews FE, Barnes DE, Yaffe K, Brayne C. Potential for primary prevention of Alzheimer's disease: an analysis of population-based data. Lancet Neurol. 13(8), 788-794 (2014).

3. De Bruijn RF, Bos MJ, Portegies ML et al. The potential for prevention of dementia across two decades: the prospective, population-based Rotterdam Study. BMC Med. 13, 132 (2015).

4. Stephan BCM, Kurth T, Matthews FE, Brayne C, Dufouil C. Dementia risk prediction in the population: are screening models accurate? Nat. Rev. Neurol. 6(6), 318-326 (2010).

5. Tang EY, Harrison SL, Errington L et al. Current developments in dementia risk prediction modelling: an updated systematic review. PLoS ONE 10(9), e0136181 (2015).

6. Chouraki V, Reitz C, Maury F et al. Evaluation of a genetic risk score to improve risk prediction for Alzheimer's disease. J. Alzheimers Dis. 53(3), 921-932 (2016).

7. Li J, Ogrodnik M, Devine S, Auerbach S, Wolf PA, Au R. Practical risk score for 5-, 10-, and 20-year prediction of dementia in elderly persons: Framingham Heart Study. Alzheimers Dement. doi:10.1016/j.jalz.2017.04.013 (2017) (Epub ahead of print).

8. Stephan BC, Tzourio C, Auriacombe $S$ et al. Usefulness of data from magnetic resonance imaging to improve prediction of dementia: population based cohort study. BMJ 350, h2863 (2015).

9. Seshadri S, Fitzpatrick AL, Ikram MA, Destefano AL et al. Charge Consortium; Gerad1 Consortium; Eadi1 Consortium. Genome-wide analysis of genetic loci associated with Alzheimer disease. JAMA 303(18), 1832-1840 (2010).

10. Walters K, Hardoon S, Petersen I et al. Predicting dementia risk in primary care: development and validation of the Dementia Risk Score using routinely collected data. BMC Med. 14, 6 (2016).

11. Vuoksimaa E, Rinne JO, Lindgren N, Heikkila K, Koskenvuo M, Kaprio J. Middle age self-report risk score predicts cognitive functioning and dementia in 20-40 years. Alzheimers Dement. (Amst). 4, 118-125 (2016).

12. Kivipelto M, Ngandu T, Laatikainen T, Winblad B, Soininen H, Tuomilehto J. Risk score for the prediction of dementia risk in 20 years among middle aged people: a longitudinal, population-based study. Lancet Neurol. 5(9), 735-741 (2006).

13. Exalto LG, Quesenberry CP, Barnes D, Kivipelto M, Biessels GJ, Whitmer RA. Midlife risk score for the prediction of dementia four decades later. Alzheimers Dement. 10(5), 562-570 (2014).

14. Ngandu T, Lehtisalo J, Solomon A et al. A 2 year multidomain intervention of diet, exercise, cognitive training, and vascular risk monitoring versus control to prevent cognitive decline in at-risk elderly people (FINGER): a randomised controlled trial. Lancet 385(9984), 2255-2263 (2015).

15. Sindi S, Calov E, Fokkens J et al. The CAIDE Dementia Risk Score App: the development of an evidence-based mobile application to predict the risk of dementia. Alzheimers Dement. (Amst). 1(3), 328-333 (2015).

16. Devlin E, Macaskill S, Stead M. 'We're still the same people': developing a mass media campaign to raise awareness and challenge the stigma of dementia. Int. J. Nonprofit Voluntary Sector Marketing 12, 47-58 (2007).

17. Nolan T, Dack C, Pal K et al. Br. J. Gen. Pract. 65(632), e152-e160 (2015).

18. Kim S, Sargent-Cox KA, Anstey KJ. A qualitative study of older and middle-aged adults' perception and attitudes towards dementia and dementia risk reduction. J. Adv. Nursing 71(7), 1694-1703 (2015). 
